\title{
Estudo comparativo do desempenho ocupacional entre crianças com atraso de desenvolvimento entre 3 e 5 anos de idade
}

\author{
Patrícia Isabel Candeias Fernandes, Maria Raquel Rodrigues Santana, \\ Susana Cristina Costa Pestana
}

Departamento de Saúde, Escola Superior de Saúde, Instituto Politécnico de Beja, Beja, Portugal.

\begin{abstract}
Resumo: Objetivos: O presente estudo teve como objetivo comparar o desempenho ocupacional de crianças com atraso de desenvolvimento, inseridas no Sistema Nacional de Intervenção Precoce na Infância (SNIPI) de Portugal, e crianças com desenvolvimento típico aos 3 e aos 5 anos de idade, bem como verificar em que áreas funcionais - ou seja, a autonomia pessoal, a mobilidade e a socialização -, as crianças com atraso de desenvolvimento apresentam mais dificuldades. Método: Tratou-se de um estudo descritivo comparativo, que contou com uma amostra de 40 participantes, sendo que 20 apresentam Desenvolvimento Típico (DT) e 20 apresentam diagnóstico de Atraso de Desenvolvimento (AD). Estes dois grupos foram divididos em dois subgrupos etários de 10 crianças (com 3 e com 5 anos). Resultados: Foi preenchido pelos pais o Inventário Pediátrico de Avaliação das Incapacidades, sendo que os resultados analisados revelam que o desempenho ocupacional de crianças com atraso de desenvolvimento, inseridas no SNIPI, é inferior ao das crianças com desenvolvimento típico, com 3 e 5 anos de idade, nas áreas da autonomia pessoal, mobilidade e socialização. Conclusão: É pertinente que sejam publicados mais estudos relacionados com a prática da terapia ocupacional, em Portugal, contribuindo, desta forma, para o conhecimento de profissionais e alunos desta área científica. Além disso, cada vez mais a prática baseada na evidência é uma abordagem adotada pelos profissionais de saúde, incluindo os terapeutas ocupacionais, sendo que, para tal, devem ser realizados estudos atuais sobretudo em Portugal.
\end{abstract}

Palavras-chave: Deficiências do Desenvolvimento, Desenvolvimento Infantil, Desempenho Ocupacional, Terapia Ocupacional, Intervenção Precoce.

\section{Comparative study of occupational performance in children with global developmental delay at 3 and 5 years old}

\begin{abstract}
Objectives: This study aims to compare the occupational performance of children with global developmental delay, inserted in SNIPI, and children with typical development, at 3 and 5 years of age, and to verify what functional areas children with global developmental delay feature more difficulties. Method: This is a comparative descriptive study including a sample of 40 participants, and 20 show Typical Development with 20 presenting diagnosis of Global Development Delay. These two groups were divided into two age subgroups of 10 children (aged 3 to 5 years). Results: The Inventory Pediatric Evaluation of Disability was filled out by parents and the results show that occupational performance of children with developmental delay, inserted in SNIPI is lower than those with typical development, with 3 and 5 years old, in the areas personal, mobility and socialization autonomy. Conclusion: The publication of more studies related to the practice of occupational therapy in Portugal is crucial,
\end{abstract}

Autor para correspondência: Maria Raquel Rodrigues Santana, Escola Superior de Saúde, Instituto Politécnico de Beja, Rua Pedro Soares, Campus do Instituo Politécnico de Beja, Apartado 6155, 7800-295, Beja, Portugal, e-mail: maria.santana@ipbeja.pt 
thus contributing to the knowledge of professionals and students in this scientific area. In addition, more and more evidence-based practice is an approach adopted by health professionals, including occupational therapists, and to this end it is extremely important to carry out newer studies locally in Portugal.

Keywords: Developmental Disabilities, Child Development, Occupational Performance, Occupational Therapy, Early Intervention.

\section{Introdução}

O conceito de atraso de desenvolvimento (AD), não sendo um diagnóstico, é utilizado quando uma criança náo atinge um marco de desenvolvimento esperado para a sua idade, ou seja, caracteriza qualquer problema relacionado com o desenvolvimento da criança (MCCONNELL, 1998 apud SILVA; ALBUQUERQUE, 2011).

Segundo Rydz et al. citado por Coelho e Rezende (2007), o desenvolvimento comporta quatro grandes áreas: motora (competências motoras grossas e finas); linguagem (competências de articulação, linguagem receptiva e expressiva, e uso de símbolos não verbais); cognitiva (resolução de problemas) e, por fim, social (interação e manutenção das relaçôes com os pares). Assim, sempre que existe um problema em uma ou mais áreas de desenvolvimento, considera-se que a criança apresenta $\mathrm{AD}$, verificando-se, como consequência, alteraçôes no seu desempenho ocupacional (PEDRETTI; EARLY, 2005).

Segundo Tavares et al. (2007), é nos dois primeiros anos de vida que a criança desenvolve competências, tais como gatinhar, andar, sentar e falar. A criança desenvolve-se controlando o seu corpo no sentido cefalocaudal e proximodistal. Os mesmos autores referem que, nos primeiros 3 anos de vida, a criança desenvolve capacidades cognitivas por se interessar pelo mundo que a rodeia e, também, pela sua necessidade de comunicação. É aos 3 anos que a criança começa a vestir as suas roupas sem a ajuda do adulto, gosta de desenhar e é capaz de andar de bicicleta. No nível da comunicação, a criança é capaz de construir frases com seis palavras, compreende regras gramaticais e alguns conceitos, e reproduz uma história.

Aos 5 anos de idade, a criança realiza atividades mais complexas, sendo capaz de usar a tesoura para cortar papel, apresenta um maior domínio dos talheres e é capaz de agarrar uma bola em movimento. É também nesta idade que a criança está mais sociável e deseja tomar as suas próprias decisóes. O seu vocabulário é mais rico e consegue expressar os seus sentimentos. Gosta de inventar histórias e começa a identificar algumas letras do alfabeto (DIAS; CORREIA; MARCELINO, 2013).
O desempenho ocupacional refere-se à prática de uma determinada ocupação resultante da interação entre o individuo, o contexto, o ambiente e a atividade (MARQUES; TRIGUEIRO, 2011). Segundo Pedretti e Early (2005), o desempenho ocupacional consiste na capacidade de realizar tarefas que permitam o desempenho e a participação nos diversos papéis ocupacionais do individuo.

Quando abordamos o desempenho ocupacional, é inevitável falar da terapia ocupacional, uma vez que, como profissão da área da saúde, podemos defini-la como a arte e a ciência de auxiliar indivíduos na execução das suas atividades de vida diária, que lhes sejam significativas, e de promover saúde e bem-estar através do envolvimento nas diversas ocupaçōes (CREPEAU; SCHELL; COHN, 2011).

$\mathrm{Na}$ visão da terapia ocupacional, a criança que apresenta $\mathrm{AD}$ não tem capacidade de se envolver/desempenhar uma ocupação, sendo que as suas ocupaçóes focam-se nas atividades de vida diária, no brincar e na educação. Assim, o foco da terapia ocupacional é identificar quais as causas e os fatores de risco, bem como aplicar instrumentos de avaliação que permitam verificar qual o impacto desse atraso no dia a dia da criança, delineando assim uma intervenção mais adequada (COELHO; REZENDE, 2007). É fundamental intervir precocemente para que as crianças que apresentam alteraçóes no seu desenvolvimento, futuramente possam tornar-se jovens e adultos capazes de participar de forma autônoma e independente na vida social, corrigindo, assim, as suas limitaçóes funcionais (SISTEMA..., 2015).

O Sistema Nacional de Intervenção Precoce na Infância (SNIPI), criado ao abrigo do Decreto-Lei $n^{\circ} 281 / 2009$, publicado a 6 de outubro no Diário da República, na sequência dos princípios estabelecidos na Convenção das Naçôes Unidas dos Direitos das Crianças e no âmbito do Plano de Ação para a Integraçáo de Pessoas com Deficiência ou Incapacidade, tem como missão garantir o serviço de Intervenção Precoce na Infância (IPI). O SNIPI atua através da articulação entre os Ministérios do Trabalho e da Solidariedade Social, da Saúde e da Educaçáo e Ciência, em conjunto com as famílias e a comunidade (SISTEMA..., 2015). 
Em Portugal, o Despacho Conjunto no 891/99, de 19 de outubro, define a IPI como uma medida de apoio integrado à criança e à sua família, incluindo ações de prevenção e reabilitação no âmbito da educação, da saúde e da ação social. Intervém junto a crianças de zero aos 6 anos de idade que apresentam alteraçóes ou risco de alteraçóes nas estruturas ou funçóes do corpo, e risco grave de atraso de desenvolvimento (RODRIGUES, 2011).

A terapia ocupacional favorece o desempenho funcional da criança focando-se no desenvolvimento das competências adequadas e necessárias à sua faixa etária, sendo este um trabalho com base no brincar. $O$ brincar não é encarado como uma atividade lúdica neste contexto, mas sim como um meio facilitador para atingir os objetivos (SOUZA; MARINO, 2013).

Um estudo realizado por Souza e Magalhães (2012) declara o desempenho funcional da criança como um aspecto importante no desenvolvimento da mesma. Revela a importância do aspecto motor pelo fato de ser o primeiro marco observável no desenvolvimento. Para além dos fatores biológicos, como a prematuridade, também existem fatores ambientais fundamentais no desenvolvimento da criança, tais como o contexto em que vive, a escolaridade dos pais/cuidadores, o seio familiar e as relações familiares.

Barros (2009) revela, no seu estudo, que crianças com atraso global de desenvolvimento, nascidas prematuramente, apresentam um desempenho funcional inferior quando comparadas a crianças nascidas de termo.

Também Lamônica e Picolini (2009) referem que crianças com atraso de desenvolvimento, nascidas prematuramente, tem maior dificuldade no que diz respeito à cognição, à socialização e à autonomia pessoal.

Posto isto, considera-se pertinente realizar um estudo que compare o desempenho ocupacional de crianças com $\mathrm{AD}$ e crianças com $\mathrm{DT}$, uma vez que os estudos encontrados apenas comparam o desempenho funcional das crianças nascidas prematuramente e a termo. Desta forma, surge a questão de investigação: O desempenho ocupacional das crianças com atraso de desenvolvimento é significativamente inferior ao das crianças com desenvolvimento típico, aos 3 e 5 anos de idade, em nível da autonomia pessoal, mobilidade e socialização? Para tal questão, levantam-se as seguintes hipóteses de investigação:

$\mathrm{H}^{1}$ : As crianças com atraso de desenvolvimento, aos 3 e 5 anos de idade, apresentam dificuldades no desempenho ocupacional no nível da autonomia pessoal, mobilidade e socialização comparativamente às crianças com desenvolvimento típico, com as mesmas faixas etárias.

$\mathrm{H}^{2}$ : As assimetrias no desempenho ocupacional das crianças com atraso de desenvolvimento, aos 3 e 5 anos de idade, verificam-se em todas as áreas das aptidóes funcionais (autonomia pessoal, mobilidade e socializaçáo).

\section{Método}

O tipo de estudo utilizado corresponde ao desenho de investigação considerado mais adequado para atingir o objetivo definido. Como tal e considerando o tema que se propóe estudar, optou-se por um estudo descritivo comparativo, transversal, de caráter quantitativo. O estudo descritivo tem como objetivo definir as caraterísticas de uma população e o estudo descritivo comparativo procura estabelecer diferenças entre os grupos em relação. De acordo com o mesmo autor, um estudo transversal mede a frequência de manifestação de um acontecimento numa populaçáo num dado momento (FORTIN, 2009).

Então, de acordo com Fortin (2009), existe uma classificação para cada variável, segundo o papel que essas variáveis desempenham na investigaçáo, podendo ser variáveis independentes, dependentes, de atributo e estranhas. Para este estudo, definem-se dois tipos de variáveis, isto é, variável dependente: desempenho ocupacional, e duas variáveis independentes: atraso de desenvolvimento e desenvolvimento típico.

Neste estudo, será verificado se as crianças com atraso de desenvolvimento, aos 3 e aos 5 anos de idade, inseridas no SNIPI, apresentam um desempenho ocupacional significativamente inferior ao das crianças com desenvolvimento típico, com a mesma idade, nas áreas da autonomia pessoal, mobilidade e socialização. As duas faixas etárias foram propositadamente selecionadas para diminuir o efeito diferenciado que períodos específicos impóem em áreas distintas de desenvolvimento.

A amostra referente às crianças com atraso de desenvolvimento foi recolhida nas Equipas Locais de Intervenção (ELI) da regiáo do Baixo Alentejo, nomeadamente, nas ELIs de Almodôvar e Ourique, de Castro Verde e Mértola, de Moura e Barrancos, e de Beja, Cuba, Alvito e Vidigueira; e da regiáo do Algarve, especificamente, as ELIs de Faro e de Tavira. No que diz respeito às crianças com desenvolvimento 
típico, a amostra foi recolhida nas Creches e Jardins de Infância de Cuba e Castro Verde.

Para este estudo, constituiu-se uma amostragem não probabilística acidental. De acordo com Fortin (2009), uma amostragem não probabilística é aquela que não oferece a todos os elementos da população a mesma possibilidade de ser escolhido para a amostra. Segundo o mesmo autor, a amostragem acidental é formada por indivíduos facilmente acessíveis e que correspondem aos critérios de inclusão. Desta forma, somente foram incluídas, na amostra, crianças que apresentaram as características exigidas nos critérios de inclusão.

A população-alvo é o conjunto de pessoas que satisfazem os critérios de seleção definidos previamente e que permitem fazer generalizaçôes (FORTIN, 2009). O mesmo autor afirma que a população é escolhida segundo critérios de inclusão, ou seja, para os indivíduos serem parte da amostra, é necessário apresentarem as mesmas características dos elementos da população. Assim, a população em estudo refere-se a crianças com $A D$, inseridas no SNIPI, e crianças com DT, com 3 e 5 anos de idade. Os critérios de inclusão definidos para a investigação consistem em: crianças com AD inseridas no SNIPI e com menos de um ano de intervenção realizada pelas ELIs, e crianças com DT com idade cronológica entre os 3 anos e 0 mês e 3 anos e 11 meses, e os 5 anos e 0 mês e 5 anos e 11 meses.

Na presente investigação, fizeram parte da amostra 40 participantes, sendo que $20(50,0 \%)$ apresentam DT e 20 (50,0\%) apresentam diagnóstico de AD tal como pode ser constatado na Tabela 1. Estes dois grupos foram divididos em dois subgrupos etários de 10 crianças, isto é, no grupo de crianças com AD, 10 crianças têm entre os 3 anos e 0 mês e 3 anos e 11 meses e as restantes tem entre 5 anos e 0 mês e 5 anos e 11 meses. O mesmo procedimento foi realizado no grupo de crianças com DT.

No que respeita ao gênero, 9 (45\%) crianças com DT são do gênero masculino e 11 (55\%) do gênero feminino e, das 20 crianças com $\mathrm{AD}, 14$ (70\%) são do gênero masculino e 6 (30\%) são do gênero feminino.

No âmbito da investigação, foi utilizado o Inventário Pediátrico de Avaliação das Incapacidades (IPAI), versão 1.0 , sendo a versão original o Pediatric Evaluation of Disability Inventory (PEDI), da autoria de Haley et al. (1992), que pretende avaliar crianças dos 6 meses aos 7,5 anos no nível da autonomia pessoal, mobilidade e socialização. Este instrumento encontra-se validado para a população portuguesa, por Moreira (2003). Primeiramente, o autor fez uma recolha bibliográfica acerca dos conceitos: Comportamento Adaptativo, Modelo Conceitual em terapia ocupacional e Modelo de Ocupação Humana, Aprendizagem, Desenvolvimento da criança nas áreas de ocupação entre os 6 meses e os 8 anos, Avaliação em terapia ocupacional e Medida e validaçáo de instrumentos de medida. Em seguida, procedeu à traduçáo do manual do teste, efetuada por dois tradutores que, juntamente com o autor, compararam as traduções das quais surgiu a primeira versão do IPAI traduzida para a língua portuguesa. Após a referida tradução, foi elaborada uma retrotradução, sendo esta analisada em conjunto com o autor para comparar com a versão original, para que não existisse distorção da percepçáo e do significado. Náo se encontrando alteraçōes ao significado do teste, esta versão foi mantida para aplicação à população portuguesa. Terminado este processo, o instrumento de medida

Tabela 1. Análise descritiva dos grupos de crianças com Desenvolvimento Típico (DT) e com Atraso de Desenvolvimento (AD), nas variáveis gênero e idade.

\begin{tabular}{|c|c|c|c|c|c|c|}
\hline \multicolumn{3}{|c|}{ Diagnóstico dos participantes } & \multirow{2}{*}{$\begin{array}{c}\text { Frequência } \\
9\end{array}$} & \multirow{2}{*}{$\begin{array}{c}\text { Percentual } \\
45,0\end{array}$} & \multirow{2}{*}{$\begin{array}{c}\text { Percentagem } \\
\text { válida }\end{array}$} & \multirow{2}{*}{$\begin{array}{c}\begin{array}{c}\text { Percentagem } \\
\text { acumulativa }\end{array} \\
45,0\end{array}$} \\
\hline \multirow{3}{*}{ DT } & \multirow{3}{*}{ Gênero } & Masculino & & & & \\
\hline & & Feminino & 11 & 55,0 & 55,0 & 100,0 \\
\hline & & Total & 20 & 100,0 & 100,0 & \\
\hline \multirow{3}{*}{ AD } & \multirow{3}{*}{ Gênero } & Masculino & 14 & 70,0 & 70,0 & 70,0 \\
\hline & & Feminino & 6 & 30,0 & 30,0 & 100,0 \\
\hline & & Total & 20 & 100,0 & 100,0 & \\
\hline \multirow{3}{*}{ DT } & \multirow{3}{*}{ Idade } & 3 & 10 & 50,0 & 50,0 & 50,0 \\
\hline & & 5 & 10 & 50,0 & 50,0 & 100,0 \\
\hline & & Total & 20 & 100,0 & 100,0 & \\
\hline \multirow{3}{*}{ AD } & \multirow{3}{*}{ Idade } & 3 & 10 & 50,0 & 50,0 & 50,0 \\
\hline & & 5 & 10 & 50,0 & 50,0 & 100,0 \\
\hline & & Total & 20 & 100,0 & 100,0 & \\
\hline
\end{tabular}


foi cedido a 13 profissionais de saúde envolvidos na validade de conteúdo, para os quais o autor apresentou os objetivos e a questáo aberta, solicitando as suas opinióes para verificar se o instrumento em estudo avaliava os objetivos a que se propunha. Os peritos tiveram decisão unânime pelo que se manteve a primeira versão do teste. Finalmente, o teste foi submetido a um pré-teste, tendo sido a primeira versão do IPAI distribuída a 10 Terapeutas Ocupacionais, na medida em que o autor pretendia a análise dos itens e se estes eram de fácil compreensão. Das respostas obtidas, não existiram propostas de alteraçôes no nível da linguagem, pelo que se chegou à versão final do IPAI (MOREIRA, 2003).

O IPAI encontra-se dividido em três partes. A Parte I: Aptidôes Funcionais, que avalia a competência da criança para desempenhar atividades nas áreas de autonomia pessoal (73 itens), mobilidade (59 itens) e socialização (65 itens), sendo que as pontuaçôes somadas em cada item (0 ou 1$)$, produzem três pontuaçóes totais relativas às Aptidóes Funcionais. Parte II: Assistência do Cuidador, que avalia a independência da criança para desempenhar as atividades funcionais nas mesmas três áreas: autonomia pessoal (8 itens), mobilidade (7 itens) e socialização (5 itens), avaliando numa escala ordinal de 0 a 5 (o primeiro score refere que a criança é totalmente dependente e o último refere que a criança é independente). Parte III: Adaptação, que informa sobre as modificaçôes necessárias para o desempenho das atividades funcionais, nas mesmas três áreas supramencionadas. Para este estudo, apenas foram utilizadas as duas primeiras partes. Este instrumento é administrado por intermédio de uma entrevista ao familiar/cuidador, com a duração aproximada de 45 a 60 minutos (MOREIRA, 2003).

Numa fase inicial, foram realizados os contatos com as várias entidades e instituiçôes a fim de obter autorização para proceder à recolha de dados. Posteriormente, foram entregues aos responsáveis (educadoras de infância e técnicos das ELIs, especificamente ao gestor de caso) o consentimento informado, bem como o IPAI, para ser preenchido pelos pais/cuidadores da criança, sendo realizada uma explicação prévia acerca da aplicação do instrumento de avaliação.

Após a obtenção dos dados, procedeu-se ao tratamento dos mesmos, com recurso ao programa estatístico Statistical Package for Social Sciences (SPSS), versão 21, sendo criada uma base de dados.

No que se refere ao tratamento estatístico, utilizaram-se aplicaçóes da estatística descritiva para verificar se existem diferenças significativas no nível das aptidóes funcionais, entre crianças com
$\mathrm{AD}$ e com DT, aos 3 e aos 5 anos de idade, bem como verificar em que áreas das aptidóes funcionais se constatam maiores dificuldades nas duas faixas etárias. Quanto à estatística descritiva, recorreu-se às frequências absolutas e relativas, e às medidas de dispersão (máximo, mínimo e desvio padrão).

A apresentaçáo dos resultados fez-se por meio de tabelas e sua respectiva descrição, de forma a facilitar a leitura e compreensão dos mesmos.

\section{Resultados e Discussão}

O tratamento dos dados recolhidos fez-se por meio de análise descritiva, através do programa de estatística SPSS, versão 21.

No que diz respeito à Parte I, relativa às Aptidóes Funcionais, são apresentados os resultados da análise descritiva para comparação dos grupos de crianças com DT e com AD, aos 3 e aos 5 anos de idade, no nível da Autonomia Pessoal, Mobilidade e Socialização. Assim, tal como consta na Tabela 2, no nível da Autonomia Pessoal, as crianças com AD com 3 anos apresentam um desempenho ocupacional inferior relativamente às crianças com DT, ainda que esta diferença represente apenas 20\% do total daquele subgrupo. No que concerne à área da Mobilidade, verifica-se que as crianças com $\mathrm{AD}$, com 3 anos, apresentam um desempenho superior, correspondente a $60 \%$ de Baixa Capacidade, comparativamente às da mesma idade com DT. Não obstante, é pertinente verificar que, daquelas, $40 \%$ não revelam dificuldades no nível da mobilidade. Relativamente à área da Socialização, constata-se que $60 \%$ das crianças com $\mathrm{AD}$, com 3 anos, revelam menor capacidade relativamente às crianças com $\mathrm{DT}$, cujo valor percentual corresponde a $100 \%$.

A Tabela 3 apresenta o resultado da análise descritiva para comparação dos grupos de crianças com DT e com AD, com 5 anos de idade, nas Aptidōes Funcionais de Autonomia Pessoal, Mobilidade e Socializaçáo. No que diz respeito à Autonomia Pessoal, verifica-se uma percentagem considerável de baixo desempenho (80\%) nas crianças com $\mathrm{AD}$, comparativamente às crianças com DT. Quanto à área da Mobilidade, importa destacar que $80 \%$ das crianças com $\mathrm{AD}$ apresentam um menor desempenho, sendo que, destas, 20\% revelam um grau de capacidade ajustado à sua faixa etária. É importante referir ainda que uma criança $(10 \%)$ com DT apresenta um baixo desempenho nesta área relativamente ao que seria de se esperar para a sua faixa etária. Relativamente às crianças com $\mathrm{AD}$ na faixa dos 5 anos de idade, verifica-se um baixo desempenho (90\%) na área 
Tabela 2. Resultado da análise descritiva para comparação dos grupos de crianças com Desenvolvimento Típico (DT) e com Atraso de Desenvolvimento (AD), com 3 anos de idade, nas aptidões funcionais de autonomia pessoal, mobilidade e socialização.

\begin{tabular}{|c|c|c|c|c|c|c|}
\hline \multicolumn{3}{|c|}{ Autonomia Pessoal } & \multirow{2}{*}{$\begin{array}{c}\text { Frequência } \\
10\end{array}$} & \multirow{2}{*}{$\begin{array}{c}\text { Percentual } \\
50,0\end{array}$} & \multirow{2}{*}{$\begin{array}{c}\text { Perc. válida } \\
100,0\end{array}$} & \multirow{2}{*}{$\begin{array}{r}\begin{array}{r}\text { Perc. } \\
\text { acum }\end{array} \\
100,0\end{array}$} \\
\hline \multirow{3}{*}{ DT } & Válido & Capacidade Normal & & & & \\
\hline & & & 10 & 50,0 & & \\
\hline & Total & & 20 & 100,0 & & \\
\hline \multirow{5}{*}{ AD } & Válido & Baixa Capacidade & 2 & 10,0 & 20,0 & 20,0 \\
\hline & & Capacidade Normal & 8 & 40,0 & 80,0 & 100,0 \\
\hline & & Total & 10 & 50,0 & 100,0 & \\
\hline & & & 10 & 50,0 & & \\
\hline & Total & & 20 & 100,0 & & \\
\hline \multicolumn{7}{|c|}{ Mobilidade } \\
\hline \multirow{3}{*}{ DT } & Válido & Capacidade Normal & 10 & 50,0 & 100,0 & 100,0 \\
\hline & & & 10 & 50,0 & & \\
\hline & Total & & 20 & 100,0 & & \\
\hline \multirow{5}{*}{ AD } & Válido & Baixa Capacidade & 6 & 30,0 & 60,0 & 60,0 \\
\hline & & Capacidade Normal & 4 & 20,0 & 40,0 & 100,0 \\
\hline & & Total & 10 & 50,0 & 100,0 & \\
\hline & & & 10 & 50,0 & & \\
\hline & Total & & 20 & 100,0 & & \\
\hline \multicolumn{7}{|c|}{ Socialização } \\
\hline \multirow{3}{*}{ DT } & Válido & Capacidade Normal & 10 & 50,0 & 100,0 & 100,0 \\
\hline & & & 10 & 50,0 & & \\
\hline & Total & & 20 & 100,0 & & \\
\hline \multirow{6}{*}{ AD } & Válido & Baixa Capacidade & 6 & 30,0 & 60,0 & 60,0 \\
\hline & & Capacidade Normal & 4 & 20,0 & 40,0 & 100,0 \\
\hline & & & & & & \\
\hline & Total & & 10 & 50,0 & 100,0 & \\
\hline & & & 10 & 50,0 & & \\
\hline & Total & & 20 & 100,0 & & \\
\hline
\end{tabular}

da Socialização, uma vez que, destas, apenas uma (10\%) apresenta um grau de desempenho adequado à sua idade, comparativamente às crianças com DT, na mesma faixa etária.

Relativamente à Parte II do IPAI, referente à Assistência do Cuidador, pretendeu-se analisar a perspectiva dos pais de crianças com $\mathrm{AD}$ com 3 e 5 anos, relativamente ao grau de assistência do cuidador no âmbito da autonomia pessoal, mobilidade e socialização. Da análise apresentada na Tabela 4, verifica-se que é necessária bastante assistência por parte do cuidador no nível da mobilidade nas crianças com 3 anos. Por outro lado, constata-se que, no âmbito da socializaçáo, o total de crianças com $\mathrm{AD}$ (10) não necessita de assistência diferenciada por parte do cuidador, comparativamente às crianças com DT.

A Tabela 5 apresenta o resultado da análise relativamente ao grau de assistência por parte do cuidador, nos dois subgrupos, com 5 anos de idade, em nível da autonomia pessoal, mobilidade e socialização. Verifica-se, assim, que as crianças com $\mathrm{AD}$ necessitam de um grau de assistência superior por parte do cuidador no nível da mobilidade, sendo que, nas áreas de autonomia pessoal e socialização, não são apresentadas diferenças significativas.

A Tabela 6 descreve os resultados da análise descritiva, sendo analisada a média para verificar em que área das aptidóes funcionais as crianças com AD, com 3 e com 5 anos de idade, evidenciam menor desempenho. Após a análise da Tabela, não se verificam diferenças significativas nas áreas de autonomia pessoal, mobilidade e socialização, quer nas crianças com 3 anos quer nas crianças com 5 anos, com $\mathrm{AD}$. Contudo, evidenciam-se valores mais baixos no nível das áreas da Mobilidade e Socialização $(M=1,40)$ nas crianças com 3 anos. Quanto às crianças com 5 anos, foram observados valores mais baixos na área da socialização $(M=1,10)$, não sendo um valor significativo. Importa realçar 
que as crianças com $\mathrm{AD}$, com 3 anos, apresentam maior grau de capacidade em nível da autonomia pessoal, tal como indica o valor apresentado na Tabela $(\mathrm{M}=1,80)$.
Perante os resultados obtidos em estudo, verifica-se que existe efetivamente uma diferença no desempenho ocupacional entre crianças com DT e com $\mathrm{AD}$, com 3 e com 5 anos de idade. No entanto,

Tabela 3. Resultado da análise descritiva para comparação dos grupos de crianças com Desenvolvimento Típico (DT) e com Atraso de Desenvolvimento (AD), com 5 anos de idade, nas aptidões funcionais de autonomia pessoal, mobilidade e socialização.

\begin{tabular}{|c|c|c|c|c|c|c|}
\hline \multicolumn{3}{|c|}{ Autonomia Pessoal } & \multirow{2}{*}{$\begin{array}{c}\text { Frequência } \\
10\end{array}$} & \multirow{2}{*}{$\begin{array}{c}\text { Percentual } \\
50,0\end{array}$} & \multirow{2}{*}{$\begin{array}{c}\text { Perc. válida } \\
100,0\end{array}$} & \multirow{2}{*}{$\begin{array}{c}\text { Perc. acum. } \\
100,0\end{array}$} \\
\hline \multirow{3}{*}{ DT } & Válido & Capacidade Normal & & & & \\
\hline & & & 10 & 50,0 & & \\
\hline & Total & & 20 & 100,0 & & \\
\hline \multirow{5}{*}{$\mathrm{AD}$} & Válido & Baixa Capacidade & 8 & 40,0 & 80,0 & 80,0 \\
\hline & & Capacidade Normal & 2 & 10,0 & 20,0 & 100,0 \\
\hline & & Total & 10 & 50,0 & 100,0 & \\
\hline & & & 10 & 50,0 & & \\
\hline & Total & & 20 & 100,0 & & \\
\hline \multicolumn{7}{|c|}{ Mobilidade } \\
\hline \multirow{5}{*}{ DT } & Válido & Baixa Capacidade & 1 & 5,0 & 10,0 & 10,0 \\
\hline & & Capacidade Normal & 9 & 45,0 & 90,0 & 100,0 \\
\hline & & Total & 10 & 50,0 & 100,0 & \\
\hline & & & 10 & 50,0 & & \\
\hline & Total & & 20 & 100,0 & & \\
\hline \multirow{5}{*}{$\mathrm{AD}$} & Válido & Baixa Capacidade & 8 & 40,0 & 80,0 & 80,0 \\
\hline & & Capacidade Normal & 2 & 10,0 & 20,0 & 100,0 \\
\hline & & Total & 10 & 50,0 & 100,0 & \\
\hline & & & 10 & 50,0 & & \\
\hline & Total & & 20 & 100,0 & & \\
\hline \multicolumn{7}{|c|}{ Socialização } \\
\hline \multirow{3}{*}{ DT } & Válido & Capacidade Normal & 10 & 50,0 & 100,0 & 100,0 \\
\hline & & & 10 & 50,0 & & \\
\hline & Total & & 20 & 100,0 & & \\
\hline \multirow{5}{*}{$\mathrm{AD}$} & Válido & Baixa Capacidade & 9 & 45,0 & 90,0 & 90,0 \\
\hline & & Capacidade Normal & 1 & 5,0 & 10,0 & 100,0 \\
\hline & & Total & 10 & 50,0 & 100,0 & \\
\hline & & & 10 & 50,0 & & \\
\hline & Total & & 20 & 100,0 & & \\
\hline
\end{tabular}

Tabela 4. Resultado da análise descritiva para comparação de grupos de crianças com Desenvolvimento Típico (DT) e com Atraso de Desenvolvimento (AD), com 3 anos, em nível da Assistência do Cuidador nas áreas de Autonomia Pessoal, Mobilidade e Socialização.

\begin{tabular}{|c|c|c|c|c|}
\hline & & \multicolumn{2}{|c|}{$\begin{array}{c}\text { Diagnóstico dos } \\
\text { participantes }\end{array}$} & \multirow[t]{2}{*}{ Total } \\
\hline & & DT & $\mathrm{AD}$ & \\
\hline \multirow{2}{*}{ Autonomia Pessoal } & Muita Assistência & 0 & 1 & 1 \\
\hline & Assistência adequada à idade da criança & 10 & 9 & 19 \\
\hline Total & & 10 & 10 & 20 \\
\hline \multirow{2}{*}{ Mobilidade } & Muita Assistência & 1 & 5 & 6 \\
\hline & Assistência adequada à idade da criança & 9 & 5 & 14 \\
\hline Total & & 10 & 10 & 20 \\
\hline \multirow{2}{*}{ Socialização } & Assistência adequada à idade da criança & 9 & 10 & 19 \\
\hline & Nenhuma Assistência & 1 & 0 & 1 \\
\hline Total & & 10 & 10 & 20 \\
\hline
\end{tabular}


Tabela 5. Resultado da análise descritiva para comparação de grupos de crianças com Desenvolvimento Típico (DT) e com Atraso de Desenvolvimento (AD), com 5 anos, em nível da Assistência do Cuidador nas áreas de Autonomia Pessoal, Mobilidade e Socialização.

\begin{tabular}{|c|c|c|c|c|}
\hline & \multicolumn{2}{|c|}{ Diagnóstico dos participantes } & \multirow{2}{*}{ Total } \\
\hline & & DT & $\mathrm{AD}$ & \\
\hline \multirow[b]{2}{*}{ Autonomia Pessoal } & Muita Assistência & 0 & 4 & 4 \\
\hline & $\begin{array}{l}\text { Assistência adequada à } \\
\text { idade da criança }\end{array}$ & 10 & 6 & 16 \\
\hline \multirow[t]{2}{*}{ Total } & & 10 & 10 & 20 \\
\hline & Muita Assistência & 4 & 6 & 10 \\
\hline Mobilidade & $\begin{array}{l}\text { Assistência adequada à } \\
\text { idade da criança }\end{array}$ & 6 & 4 & 10 \\
\hline \multirow[t]{2}{*}{ Total } & & 10 & 10 & 20 \\
\hline & Muita Assistência & 0 & 2 & 2 \\
\hline Socialização & $\begin{array}{l}\text { Assistência adequada à } \\
\text { idade da criança }\end{array}$ & 10 & 8 & 18 \\
\hline Total & & 10 & 10 & 20 \\
\hline
\end{tabular}

Tabela 6. Resultados da análise descritiva para verificação do grau de incapacidade das crianças com Atraso de Desenvolvimento (AD), aos 3 e 5 anos de idade, nas Aptidões Funcionais da Autonomia Pessoal, Mobilidade e Socialização.

\begin{tabular}{llcccc}
\hline \multicolumn{1}{c}{ Diagnóstico dos participantes } & N & Mínimo & Máximo & Média & Desvio padrão \\
\hline $\begin{array}{l}\text { Parte I: Aptidões Funcionais } \\
\text { Autonomia Pessoal 3 anos }\end{array}$ & 10 & 1 & 2 & 1,80 & 0,422 \\
$\quad \begin{array}{l}\text { Parte I: Aptidões Funcionais } \\
\text { Mobilidade 3 anos }\end{array}$ & 10 & 1 & 2 & 1,40 & 0,516 \\
$\begin{array}{l}\text { Parte I: Aptidões Funcionais } \\
\text { Socialização 3 anos }\end{array}$ & 10 & 1 & 2 & 1,40 & 0,516 \\
$\begin{array}{l}\text { Parte I: Aptidões Funcionais } \\
\text { Autonomia Pessoal 5 anos }\end{array}$ & 10 & 1 & 2 & 1,20 & 0,422 \\
$\quad \begin{array}{l}\text { Parte I: Aptidões Funcionais } \\
\text { Mobilidade 5 anos }\end{array}$ & 10 & 1 & 2 & 1,20 & 0,422 \\
$\quad \begin{array}{l}\text { Parte I Aptidões Funcionais } \\
\text { Socialização 5 anos }\end{array}$ & 10 & 1 & 2 & 1,10 & 0,316 \\
$\quad$ N válido (de lista) & 0 & & & & \\
\hline
\end{tabular}

o número total da amostra não é significativo para a reproduçáo de novos estudos. Barros (2009) revela, no seu estudo, que as crianças prematuras com atraso de desenvolvimento apresentam um desempenho funcional inferior, em nível da autonomia pessoal, mobilidade e socializaçáo, quando comparadas com crianças com desenvolvimento típico. Posto isto, tal como o autor anteriormente referenciado, o estudo desenvolvido pela pesquisadora vai ao encontro dos resultados obtidos; no entanto, o presente estudo centrou-se em crianças com AD independentemente de os participantes deste grupo serem prematuros.

Os resultados apresentados evidenciam um grau de incapacidade mais elevado nas áreas da mobilidade e socializaçáo, no que diz respeito às crianças com 3 anos, com $\mathrm{AD}$, do que na área de autonomia pessoal. Quanto à faixa etária dos
5 anos, estas apresentam um grau de incapacidade significativamente inferior em nível da autonomia pessoal e mobilidade, comparativamente às crianças com DT. No entanto, importa referir que a área da socialização destaca-se por ser aquela em que as crianças com 5 anos e com $\mathrm{AD}$ demonstraram um desempenho inferior relativamente às outras áreas em estudo. Segundo um estudo realizado por Lamônica e Picolini (2009), as crianças com atraso de desenvolvimento apresentam maior dificuldade no nível das áreas da socialização e autonomia pessoal. Embora tal conclusão vá ao encontro dos resultados apresentados no nosso estudo, importa referir que aqueles autores estudaram crianças com atraso de desenvolvimento associado à prematuridade.

Importa referir que, no nível da assistência do cuidador, verifica-se que as crianças com 3 anos, 
com $\mathrm{AD}$, somente necessitam de mais ajuda por parte do cuidador no nível da mobilidade. Quanto às áreas de autonomia pessoal e socializaçấo, não se constata a necessidade de assistência por parte do cuidador. Também no subgrupo de crianças com $\mathrm{AD}$, com 5 anos de idade, verificou-se que necessitam de mais ajuda do cuidador em nível de mobilidade, contrariamente às áreas da autonomia pessoal e socialização.

Assim, os resultados do estudo espelham que as crianças com 3 anos, com $\mathrm{AD}$, apresentam um menor grau de desempenho nas áreas da mobilidade e socialização, enquanto as crianças com 5 anos, com $\mathrm{AD}$, revelam um menor desempenho em nível da autonomia pessoal, mobilidade e socialização, sendo que a última representa o valor mais elevado. Considera-se pertinente referir que, através da análise dos resultados obtidos, constata-se que à medida que a criança cresce, são notórias maiores dificuldades no nível do desempenho ocupacional.

\section{Conclusão}

Após o término do estudo, é possível responder à questão de investigação, ou seja: $\mathrm{O}$ desempenho ocupacional das crianças com atraso de desenvolvimento, inseridas no SNIPI, é inferior ao das crianças com desenvolvimento típico, com 3 e 5 anos de idade, nas áreas da autonomia pessoal, mobilidade e socialização?

Perante os resultados apresentados, verifica-se que existe efetivamente uma diferença no que se refere ao desempenho ocupacional entre crianças com $\mathrm{AD}$ e crianças com DT, sendo que as primeiras apresentam um desempenho ocupacional inferior quando comparadas com as segundas. É possível concluir, também, que as áreas da mobilidade e socialização são aquelas em que as crianças com $\mathrm{AD}$, com 3 anos, apresentam um grau de incapacidade mais elevado, o que conduz a um desempenho ocupacional inferior comparativamente às crianças com DT. Por outro lado, e analisando-se os resultados, constata-se que, no que diz respeito às crianças com AD com 5 anos de idade, o desempenho ocupacional é inferior, comparativamente, às crianças com DT, uma vez que grande percentagem de crianças apresenta um baixo grau de capacidade em todas as áreas das aptidôes funcionais, especialmente na área da socialização. Pode também verificar-se que, ao longo do crescimento da criança, são notórias mais dificuldades no desempenho ocupacional, sendo que se torna evidente um grau de incapacidade mais elevado em todas as áreas das aptidóes funcionais, o que não se verifica aos 3 anos de idade. Posto isto, a pesquisadora considera pertinente a realização de novos estudos, no sentido de comprovar tal apreciação.

Em relação à segunda parte do IPAI, verifica-se que não existe coerência entre as respostas submetidas na primeira e na segunda parte do questionário, nomeadamente no nível dos 3 anos, uma vez que, segundo a percepção dos pais, as crianças não necessitam de muita assistência por parte do cuidador, embora apresentem um baixo desempenho nas áreas de mobilidade e socialização.

A pesquisadora considera que tais resultados não assumem um carácter significativo, pelo reduzido número de amostra. Desta forma, sugere a realização de novos estudos, desenvolvidos com mais rigor, tal como a investigaçáo deve merecer. Outra razão diz respeito ao fato de os pais das crianças em estudo não terem respondido a todos os itens relacionados com os dados biográficos, motivo pelo qual não pode ser feita uma análise mais detalhada dos resultados nomeadamente da relaçáo entre a escolaridade dos pais e o $\mathrm{AD}$.

\section{Referências}

BARROS, M. L. Desempenho funcional no auto-cuidado, mobilidade e função social aos 12 meses de vida em crianças nascidas permaturamente. 2009. 68 f. Dissertação (Mestrado em Saúde Materno Infantil) - Instituto de Medicina Integral Profo Fernando Figueira, Recife, 2009.

COElHo, Z. A.; REZENDE, M. B. Atraso no Desenvolvimento. In: CAVALCANTI, A.; GALVÃO, C. Terapia Ocupacional: fundamentação \& prática. Rio de Janeiro: Guanabara Koogan, 2007. p. 229-307.

CREPEAU, E. B.; SCHELL, B. A.; COHN, E. Prática de Terapia Ocupacional Contemporânea nos Estados Unidos. In: CREPEAU, E. B.; COHN, E. S.; SCHELL, B. A. Williard \& Spackman: Terapia Ocupacional. Rio de Janeiro: Guanabara Koogan, 2011. p. 219-223.

DIAS, I. S.; CORREIA, S.; MARCELINO, P. Desenvolvimento na primeira infância: características valorizadas pelos futuros educadores de infância. Revista Eletrônica de Educação, São Carlos, v. 7, n. 3, p. 9-24, 2013.

FORTIN, M. F. Fundamentos e etapas do processo de investigação. Lisboa: Lusodidacta, 2009

LAMÔNICA, D. C.; PICOLINI, M. M. Habilidades do desenvolvimento de prematuros. Revista CEFAC, São Paulo, v. 11, p. 145-153, 2009. Suplemento 2.

MARQUES, A.; TRIGUEIRO, M. J. Enquadramento da prática da Terapia Ocupacional: domínio \& processo. Port: Livpsic, 2011.

MOREIRA, N. Inventário Pediátrico de Avaliação das Incapacidades. Monografia (Graduação em Terapia Ocupacional)-Escola Superior de Saúde do Alcoitão, Lisboa, 2003. 
PEDRETTI, L. W.; EARLY, M. B. Terapia Ocupacional: capacidades práticas para as disfunções físicas. São Paulo: ROCA, 2005.

RODRIGUES, M. G. As perceçóes dos educadores de infância sobre a intervenção precoce. 2011. 71 f. Dissertação (Mestrado em Psicologia da Educação) - Universidade da Madeira, Portugal, 2011.

SILVA, E.; AlBUQUERQUE, C. P. Atraso do desenvolvimento: a imprecisão de um termo. Psicologia, Saúde e Doenças, Portugal, v. 12, n. 1, p. 19-39, 2011.

SISTEMA NACIONAL DE INTERVENÇÃO PRECOCE NA INFÂNCIA - SNIPI. Portugal. Disponível em: <http://www.dgs.pt/ms/12/default.aspx?id=5525>. Acesso em: 8 jan. 2015.

SOUZA, A. C.; MARINO, M. S. Atuação do Terapeuta Ocupacional com criança com atraso neuropsicomotor. Cadernos de Terapia Ocupacional da UFSCar, São Carlos, v. 21, n. 1, p. 149-153, 2013.

SOUZA, E. S.; MAGALHÂES, L. C. Desenvolvimento motor e funcional em crianças nascidas pré-termo e a termo: influência de fatores de risco biológico e ambiental. Revista Paulista de Pediatria, Belo Horizonte, v. 30, n. 4, p. 462-70, 2012.

TAVARES, J. et al. Manual de psicologia do desenvolvimento. Porto: Porto Editora, 2007.

\section{Contribuição dos Autores}

Patrícia Isabel Candeias Fernandes: foi responsável pela conceção e redação da presente investigação. Maria Raquel Rodrigues Santana: foi orientadora da investigação. Susana Cristina Costa Pestana: foi coorientadora da investigação. Todas as autoras aprovaram a versão final do artigo. 